\title{
@creative
commons
}

ISSN 1855-3966 (printed edn.), ISSN 1855-3974 (electronic edn.)

ARS MATHEMATICA CONTEMPORANEA 19 (2020) 231-247

https://doi.org/10.26493/1855-3974.2125.7b0

(Also available at http://amc-journal.eu)

\section{On resolving sets in the point-line incidence graph of $\mathrm{PG}(n, q)$}

\author{
Daniele Bartoli * \\ Dipartimento di Matematica e Informatica, Università degli Studi di Perugia, \\ Via Vanvitelli 1, 06123 Perugia, Italy \\ György Kiss ${ }^{\dagger}$ \\ Department of Geometry and MTA-ELTE Geometric and Algebraic \\ Combinatorics Research Group Eötvös Loránd University, \\ Budapest, 1117 Budapest, Pázmány Péter sétány 1/C, Hungary, and \\ FAMNIT, University of Primorska, 6000 Koper, Glagoljaška 8, Slovenia

\section{Stefano Marcugini $\ddagger\left(\mathbb{D}\right.$, Fernanda Pambianco ${ }^{\ddagger}$} \\ Dipartimento di Matematica e Informatica, Università degli Studi di Perugia, \\ Via Vanvitelli 1, 06123 Perugia, Italy
}

Received 25 September 2019, accepted 13 July 2020, published online 15 November 2020

\begin{abstract}
Lower and upper bounds on the size of resolving sets and semi-resolving sets for the point-line incidence graph of the finite projective space $\mathrm{PG}(n, q)$ are presented. It is proved that if $n>2$ is fixed, then the metric dimension of the graph is asymptotically $2 q^{n-1}$.

Keywords: Point-line incidence graph, resolving sets, finite projective spaces.

Math. Subj. Class. (2020): 05B25, 05C12, 51E20

* The research was supported by the Italian National Group for Algebraic and Geometric Structures and their Applications (GNSAGA - INDAM).

${ }^{\dagger}$ Corresponding author. The research was supported by the Hungarian National Research Development and Innovation Office, OTKA grant no. K 124950, and by the Slovenian Research Agency (research project J1-9110).

¥ The research was supported by the Italian National Group for Algebraic and Geometric Structures and their Applications (GNSAGA - INDAM), and by the University of Perugia (Project: "Curve, codici e configurazioni di punti", Base Research Fund 2018).

E-mail addresses: daniele.bartoli@unipg.it (Daniele Bartoli), kissgy@cs.elte.hu (György Kiss), stefano.marcugini@unipg.it (Stefano Marcugini), fernanda.pambianco@unipg.it (Fernanda Pambianco)
\end{abstract}




\section{Introduction}

For a simple, connected, finite graph $\Gamma=(V, E)$ and $x, y \in V$ let $d(x, y)$ denote the length of a shortest path joining $x$ and $y$.

Definition 1.1. Let $\Gamma=(V, E)$ be a finite, connected, simple graph. Two vertices $v_{1}, v_{2} \in$ $V$ are divided by $S=\left\{s_{1}, s_{2}, \ldots, s_{r}\right\} \subset V$ if there exists $s_{i} \in S$ so that $d\left(v_{1}, s_{i}\right) \neq$ $d\left(v_{2}, s_{i}\right)$. A vertex $v \in V$ is resolved by $S$ if the ordered sequence $\left(d\left(v, s_{1}\right), d\left(v, s_{2}\right), \ldots\right.$, $\left.d\left(v, s_{r}\right)\right)$ is unique. $\mathcal{S}$ is a resolving set in $\Gamma$ if it resolves all the elements of $V$. The metric dimension of $\Gamma$, denoted by $\mu(\Gamma)$, is the size of the smallest example of resolving set in it.

The study of metric dimension is an interesting problem in its own right and it is also motivated by the connection with the base size of the corresponding graph. The base size of a permutation group is the smallest number of points whose stabilizer is the identity. The base size of $\Gamma$, denoted by $b(\Gamma)$, is the base size of its automorphism group $\operatorname{Aut}(\Gamma)$. The study of base size dates back more than 50 years, see [18]. A resolving set in $\Gamma$ is obviously a base for $\operatorname{Aut}(\Gamma)$, so the metric dimension of a graph gives an upper bound on its base size. The difference $\mu(\Gamma)-b(\Gamma)$ is called the dimension jump of $\Gamma$. Distance-transitive graphs whose dimension jump is large with respect to the number of vertices are rare, and hence interesting objects. For more information about general results on metric dimension and base size we refer the reader to the survey paper of Bailey and Cameron [2].

Resolving sets for incidence graphs of some linear spaces were investigated by several authors $[4,9,10,12]$. In these cases much better bounds than the general ones are known. Estimates on the size of blocking sets can be used to prove lower bounds on the metric dimension, and the knowledge of geometric properties is useful for constructions and upper bounds. It was shown by Héger and Takáts [12] that the metric dimension of the point-line incidence graph of a projective plane of order $q$ is $4 q-4$ if $q \geq 23$. In a recent paper Héger et al. [11] extended this result for small values of $q$, too.

There are two natural generalizations of this planar result in higher dimensional spaces: one can consider either the point-hyperplane incidence graph, or the point-line incidence graph of $\mathrm{PG}(n, q)$. In the former case resolving sets are connected with lines in a higgledypiggledy arrangement which were investigated by Fancsali and Sziklai [9]. Their results were recently improved by the authors of this paper [5]. The latter case is studied in the present paper. We assume that the reader is familiar with finite projective geometries. For a detailed description of these spaces we refer to [14, 16].

Let $\Gamma_{n, q}$ denote the point-line incidence graph of the finite projective space PG $(n, q)$. The two sets of vertices of this bipartite graph correspond to points and lines of $\operatorname{PG}(n, q)$, respectively, and there is an edge between two vertices if and only if the corresponding point is incident with the corresponding line. In $\Gamma_{n, q}$ the distance of two different lines is 2 if they intersect each other and 4 if they are skew. The distance of a point $P$ and a line $\ell$ is 1 if $P$ is on $\ell$, and it is 3 if $P$ is not on $\ell$. Finally, the distance of two different points is always 2 . Hence, points cannot be resolved by other points. Considering these properties, the following definitions are natural.

Definition 1.2. A set $\mathcal{S}$ of points and lines of $\operatorname{PG}(n, q)$ is a semi-resolving set for points (lines) in $\Gamma_{n, q}$ if it resolves all the vertices of $\Gamma_{n, q}$ corresponding to points (lines).

Definition 1.3. Let $\mathcal{S}$ be a (semi-)resolving set in $\Gamma_{n, q}$. A point or a line is called inner (outer) if it is (not) in $\mathcal{S}$. An outer point is called $t$-covered if it incident with exactly $t$ 
lines of $\mathcal{S}$. A point or a line is called uncovered if it has empty intersection with all elements of $\mathcal{S}$.

The paper is organized as follows. First, in Section 2 we provide a pure combinatorial proof of a lower bound for resolving sets in $\Gamma_{n, q}$. In the second part of the section interesting constructions are presented for $n \geq 3$ which yield examples asymptotically close to the lower bound. These resolving sets are related with regular spreads of lines in projective spaces. We prove that the metric dimension of $\Gamma_{n, q}$ is asymptotically $2 q^{n-1}$ and its dimension jump is roughly $2 \sqrt{v}$ where $v$ denotes the number of its vertices. In Section 3 algebraic curves and blocking sets are applied. We consider a different type of line spread in $\operatorname{PG}(3, q)$ and we obtain examples of resolving sets in $\Gamma_{n, q}$ of smaller size when $q=p^{h}$, $p$ prime and $h>1$. Finally, in Section 4 computer aided results for small values of $q$ are given.

\section{General bounds}

In this section we present lower and upper bounds on $\mu\left(\Gamma_{n, q}\right)$ for all $q$ and $n \geq 3$. Particular attention is paid to the case $n=3$, since general upper bounds in any dimension depend on the 3-dimensional upper bound, see Proposition 2.15 and Theorem 2.11.

Theorem 2.1. The size of any semi-resolving set for points in $\Gamma_{n, q}$ is at least

$$
2 \frac{q^{n+1}-q}{q^{2}+q-2} .
$$

Proof. Let $\mathcal{S}$ be a semi-resolving set for points in $\Gamma_{n, q}$ which consists of $k$ lines and $m$ points.

Count in two different ways the number of incident point-line pairs $(P, \ell)$ with $\ell \in \mathcal{S}$. On the one hand, this number is exactly $k(q+1)$. On the other hand, there is at most one uncovered point, the number of 1-covered points is at most $k$ and any other outer point must be covered by at least two lines of $\mathcal{S}$. Hence

$$
k(q+1) \geq k+2\left(\frac{q^{n+1}-1}{q-1}-1-k-m\right),
$$

so

$$
k+m \geq \frac{2\left(q^{n+1}-q\right)}{(q-1)(q+2)}+\frac{q m}{q+2} .
$$

This gives the required inequality at once.

Corollary 2.2. The size of any resolving set in $\Gamma_{3, q}$ is at least $2\left(q^{2}-q+3-\frac{6}{q+2}\right)$.

Corollary 2.3. The metric dimension of $\Gamma_{3, q}$ is at least $2\left(q^{2}-q+3\right)$ for $q>10$.

From now on we focus on upper bounds which will be given by constructions. For our examples we need the notion of spreads, in particular line spreads.

Definition 2.4. A $k$-spread $\mathcal{S}^{k}$ of $\operatorname{PG}(n, q)$ is a set of $k$-dimensional subspaces with the property that each point of $\mathrm{PG}(n, q)$ is incident with exactly one element of $\mathcal{S}^{k}$. 
By definition, a $k$-spread of $\operatorname{PG}(n, q)$ consists of $\frac{q^{n+1}-1}{q^{k+1}-1}$ elements. The following theorem about the existence of spreads was proved independently by several authors, see $[1,7,17]$.

Theorem 2.5. The projective space $\operatorname{PG}(n, q)$ has a $k$-spread if and only if $(k+1) \mid(n+1)$.

Hence there exists a line spread in any odd dimensional projective space. Two line spreads are said to be disjoint, if they do not share any common line. Our first construction for a semi-resolving set for points in $\Gamma_{n, q}$ is based on disjoint line spreads. We use the following theorem of Etzion [8] about the existence of disjoint line spreads.

Theorem 2.6 (Etzion). If $n \geq 3$ is odd, then there exist at least two disjoint line spreads in $\mathrm{PG}(n, q)$.

Theorem 2.7. If $n \geq 3$ is odd, then there exists a semi-resolving set for points in $\Gamma_{n, q}$ of size

$$
r_{\mathcal{P}}(n, q)=2 q^{2} \frac{q^{n-1}-1}{q^{2}-1} .
$$

Proof. Let $\mathcal{L}_{1}$ and $\mathcal{L}_{2}$ be two disjoint line spreads in $\mathrm{PG}(n, q)$, and $\ell_{i} \in \mathcal{L}_{i}$ be arbitrary lines. We claim that $\mathcal{S}=\mathcal{L}_{1} \cup \mathcal{L}_{2} \backslash\left\{\ell_{1}, \ell_{2}\right\}$ is a semi-resolving set for points in $\Gamma_{n, q}$.

Each point not in $\ell_{i}$ is contained in a unique pair of lines $\left(r_{1}, r_{2}\right) \in \mathcal{L}_{1} \times \mathcal{L}_{2}$. Each point of $\ell_{1} \backslash \ell_{2}$ is contained in a unique line of $\mathcal{L}_{1} \cup \mathcal{L}_{2} \backslash\left\{\ell_{1}, \ell_{2}\right\}$ and each point of $\ell_{2} \backslash \ell_{1}$ is contained in a unique line of $\mathcal{L}_{1} \cup \mathcal{L}_{2} \backslash\left\{\ell_{1}, \ell_{2}\right\}$. The (possible) unique point $\ell_{1} \cap \ell_{2}$ is the only point of $\operatorname{PG}(n, q)$ not contained in any line of $\mathcal{L}_{1} \cup \mathcal{L}_{2} \backslash\left\{\ell_{1}, \ell_{2}\right\}$. The size of $\mathcal{S}$ is $2 \frac{q^{n+1}-1}{q^{2}-1}-2$, hence the statement follows.

Proposition 2.8. Let $\Sigma$ be a hyperplane and $\mathcal{L}$ be a line spread in $\mathrm{PG}(n, q), n \geq 3$ odd. Then $\Sigma$ contains exactly $\frac{q^{n-1}-1}{q^{2}-1}$ elements of $\mathcal{L}$.

Proof. Any element of $\mathcal{L}$ is either fully contained in $\Sigma$, or intersects it in exactly 1 point. The elements of $\mathcal{L}$ partition the set of points of $\Sigma$. Hence, if $x$ denotes the number of fully contained lines, then

$$
\frac{q^{n}-1}{q-1}=(q+1) x+\left(\frac{q^{n+1}-1}{q^{2}-1}-x\right) .
$$

The claim follows from this equation at once.

Theorem 2.9. Let $\mathcal{L}_{1}$ be a line spread in $\mathrm{PG}(3, q)$. Then there exists another line spread $\mathcal{L}_{2}$ in $\mathrm{PG}(3, q)$ such that $\mathcal{L}_{1}$ and $\mathcal{L}_{2}$ do not share any common line.

Proof. Let $f(X, Y)$ be an irreducible homogeneous quadratic polynomial and $\mathcal{H}_{i}^{\prime}$ denote the hyperbolic quadric in $\mathrm{PG}(3, q)$ with equation

$$
f\left(X_{0}, X_{1}\right)+i f\left(X_{2}, X_{3}\right)=0
$$

for $i=1,2, \ldots, q-1$. Apply a suitable linear transformation so that the images $\ell_{1}$ and $\ell_{2}$ of the lines $\ell_{1}^{\prime}: X_{0}=X_{1}=0$ and $\ell_{2}^{\prime}: X_{2}=X_{3}=0$ do not belong to $\mathcal{L}_{1}$. Let $\mathcal{H}_{i}$ denote the image of $\mathcal{H}_{i}^{\prime}$, and let $\mathcal{E}_{i}$ and $\mathcal{F}_{i}$ denote the two reguli of lines on $\mathcal{H}_{i}$. Then for each $i$ at most one of $\mathcal{E}_{i}$ and $\mathcal{F}_{i}$ contains some elements of $\mathcal{L}_{1}$, because any line of $\mathcal{E}_{i}$ intersects 
any line of $\mathcal{F}_{i}$ and no two elements of $\mathcal{L}_{1}$ intersect each other. Hence we can choose the notation so that $\mathcal{E}_{i}$ does not contain any element of $\mathcal{L}_{1}$ for all $i$. This implies that the spread

$$
\mathcal{L}_{2}=\bigcup_{i=1}^{q-1} \mathcal{E}_{i} \cup\left\{\ell_{1}, \ell_{2}\right\}
$$

does not share any common line with $\mathcal{L}_{1}$.

The next proposition gives a useful recursive construction method.

Proposition 2.10. Let $\mathcal{S}$ be a semi-resolving set for points in $\Gamma_{d, q}$ of size $k$. Suppose that $m$ elements of $\mathcal{S}$ are contained in a hyperplane $\Sigma_{d-1}$ of $\mathrm{PG}(d, q)$, and $\Sigma_{d-1}$ also contains the (at most one) uncovered point. Then $\Gamma_{d+1, q}$ has a semi-resolving set for points of size $(q+1) k-q m$.

Moreover, if $\mathcal{S}$ is a resolving set in $\Gamma_{d, q}$ and $\Sigma_{d-1}$ also contains the (at most one) uncovered line, then $\Gamma_{d+1, q}$ admits a resolving set of size $(q+1) k-q m+\frac{q^{d-1}-1}{q-1}$.

Proof. Embed $\Sigma_{d-1} \subset \mathrm{PG}(d, q)$ into $\mathrm{PG}(d+1, q)$, and consider in $\mathrm{PG}(d+1, q)$ the pencil of hyperplanes with carrier $\Sigma_{d-1}$. These hyperplanes, $\Sigma_{d}^{1}, \Sigma_{d}^{2}, \ldots, \Sigma_{d}^{q+1}$, are isomorphic to $\operatorname{PG}(d, q)$. Take a copy of $\mathcal{S}$ in $\Sigma_{d}^{i}$ and denote it by $\mathcal{S}^{i}$ for $i=1,2, \ldots, q+1$. Finally, let

$$
\overline{\mathcal{S}}=\bigcup_{i=1}^{q+1} \mathcal{S}^{i}
$$

We claim that $\overline{\mathcal{S}}$ is a semi-resolving set for points in $\Gamma_{n+1, q}$. Inner points are resolved by definition. If two outer points, $P_{1}$ and $P_{2}$, are in the same $\Sigma_{d}^{i}$, then they are already divided by $\mathcal{S}^{i}$. If $P_{1}$ is in $\mathcal{S}^{i}$ and $P_{2}$ is in $\mathcal{S}^{j}$ with $i \neq j$, then, as none of $P_{1}$ and $P_{2}$ is uncovered and none of them is in $\Sigma_{d-1}$, there exist distinct lines $\ell^{i} \in \mathcal{S}^{i}$ through $P_{1}$ and $\ell^{j} \in \mathcal{S}^{j}$ through $P_{2}$. Hence $\ell^{i}$ does not contain $P_{2}$, so $d\left(P_{1}, \ell^{i}\right) \neq d\left(P_{1}, \ell^{i}\right)$. Since the size of $\overline{\mathcal{S}}$ is $m+(q+1)(k-m)$, the first part of the statement is proved.

Now suppose that $\mathcal{S}$ is a resolving set in $\Gamma_{d, q}$. Then the elements of any point-line pair are obviously divided by $\overline{\mathcal{S}}$. Let $\ell_{1}$ and $\ell_{2}$ be two lines. If at least one of them is an element of $\overline{\mathcal{S}}$, then they are divided by definition. From now on we assume that none of the two lines is an element of $\overline{\mathcal{S}}$. We distinguish three main cases and some subcases.

1. If both of them are entirely contained in the same $\Sigma_{d}^{i}$, then they are divided by $\mathcal{S}^{i}$.

2. If there is no $\Sigma_{d}^{i}$ that contains both $\ell_{1}$ and $\ell_{2}$, but each of the lines is entirely contained in some $\Sigma_{d}^{i}$, say $\ell_{1} \subset \Sigma_{d}^{i_{1}}$ and $\ell_{2} \subset \Sigma_{d}^{i_{2}}$, then none of the lines is in $\Sigma_{d-1}$. Let $P_{j}$ denote the unique point $\ell_{j} \cap \Sigma_{d-1}$ for $j=1,2$.

- If $P_{1}=P_{2}$, then let $P_{3} \neq P_{1}$ be a point on $\ell_{1}$. Since $\mathcal{S}^{i_{1}}$ is a semi-resolving set for points in $\Sigma_{d}^{i_{1}}$ and $P_{3}$ is not an uncovered point, either $P_{3} \in \mathcal{S}^{i_{1}}$ or there exists at least one line $\ell \in \mathcal{S}^{i_{1}}$ which contains $P_{3}$ but does not contain $P_{1}$. In the former case $d\left(\ell_{1}, P_{3}\right)=1 \neq 3=d\left(\ell_{2}, P_{3}\right)$. In the latter case $d\left(\ell_{1}, \ell\right)=2 \neq 4=d\left(\ell_{2}, \ell\right)$, so we are done.

- If $P_{1} \neq P_{2}$, then we may assume that $P_{1}$ is not an uncovered point, because there is at most one uncovered point. Again, either $P_{1} \in \mathcal{S}^{i_{1}}$ or there exists at least one line $\ell \in \mathcal{S}^{i_{1}}$ which contains $P_{1}$ but does not contain $P_{2}$. In the former case $d\left(\ell_{1}, P_{1}\right)=1 \neq 3=d\left(\ell_{2}, P_{1}\right)$, while in the latter case $d\left(\ell_{1}, \ell\right)=2 \neq$ $4=d\left(\ell_{2}, \ell\right)$, so $\ell_{1}$ and $\ell_{2}$ are divided by $\mathcal{S}^{i_{1}}$. 
3. If $\ell_{1}$ is not contained in any $\Sigma_{d}^{i}$, then it cannot meet $\Sigma_{d-1}$, so there exists a unique point $P_{1}^{i}=\ell_{1} \cap \Sigma_{d}^{i}$ for all $i=1,2, \ldots, q+1$.

- If $\ell_{2}$ is not contained in any $\Sigma_{d}^{i}$, then it cannot meet $\Sigma_{d-1}$, so there exists a unique point $P_{2}^{i}=\ell_{2} \cap \Sigma_{d}^{i}$ for all $i=1,2, \ldots, q+1$. The two lines have at most one point of intersection, hence there exist at least $q$ superscripts so that $P_{1}^{i} \neq P_{2}^{i}$. Since $\mathcal{S}^{i}$ is a semi-resolving set for points in $\Sigma_{d}^{i}$, there exists at least one element $s \in \mathcal{S}^{i}$ so that $d\left(P_{1}^{i}, s\right) \neq d\left(P_{2}^{i}, s\right)$. Hence

$$
d\left(\ell_{1}, s\right)=d\left(P_{1}^{i}, s\right)+1 \neq d\left(P_{2}^{i}, s\right)+1=d\left(\ell_{2}, s\right),
$$

so the lines are divided by $\mathcal{S}^{i}$.

- If $\ell_{2}$ is contained in a unique $\Sigma_{d}^{i}$, then it is not contained in $\Sigma_{d-1}$, so there exists a unique point $P_{2}=\ell_{2} \cap \Sigma_{d-1}$. Let $j \neq i$ and consider $\Sigma_{d}^{j}$, which contains both $P_{1}^{j}$ and $P_{2}$. Since $\mathcal{S}^{j}$ is a semi-resolving set for points in $\Sigma_{d}^{j}$, there exists at least one element $s \in \mathcal{S}^{j}$ so that $d\left(P_{1}^{j}, s\right) \neq d\left(P_{2}, s\right)$. Hence

$$
d\left(\ell_{1}, s\right)=d\left(P_{1}^{j}, s\right)+1 \neq d\left(P_{2}, s\right)+1=d\left(\ell_{2}, s\right),
$$

the claim is proved.

- Finally, suppose that $\ell_{2}$ is contained in $\Sigma_{d-1}$. Then $\ell_{1}$ and $\ell_{2}$ are not necessarily divided by $\overline{\mathcal{S}}$. Suppose that $\overline{\mathcal{S}}$ consists of lines only. Then $\ell_{2}$ and $P_{1}^{i}$ are divided by $\mathcal{S}^{i}$, but it could happen that a line of $\mathcal{S}^{i}$ intersects $\ell_{2}$ if and only if it contains $P_{1}^{i}$. If it holds for all $i$, then $\ell_{1}$ and $\ell_{2}$ have the same distance sequence with respect to $\overline{\mathcal{S}}$. We can handle this problem by extending $\overline{\mathcal{S}}$ with all the $\frac{q^{d-1}-1}{q-1}$ points of a hyperplane in $\Sigma_{d-1}$. Then $\ell_{2}$ contains at least one of these points and $\ell_{1}$ does not contain any of them. Hence the two lines are divided.

The size of the constructed resolving set is $(q+1) k-q m+\frac{q^{d-1}-1}{q-1}$, the statement is proved.

Theorem 2.11. If $n \geq 4$ is even, then there exists a semi-resolving set for points in $\Gamma_{n, q}$ of size

$$
r_{\mathcal{P}}(n, q)=2 q^{n-1}+2 q^{n-2}+2\left(q^{n-4}+q^{n-6}+\cdots+q^{2}\right) .
$$

Proof. We apply Proposition 2.10 for $d=n-1$. Let $\mathcal{S}$ be the semi-resolving set for points in $\Gamma_{n-1, q}$ which was constructed in Theorem 2.7. Its size is

$$
k=2 \frac{q^{n}-q^{2}}{q^{2}-1} .
$$

By Proposition 2.8, we can choose the hyperplane $\Sigma_{n-2}$ so that it contains

$$
m=2\left(\frac{q^{n-2}-1}{q^{2}-1}-1\right)=2 \frac{q^{n-2}-q^{2}}{q^{2}-1}
$$

elements of $\mathcal{S}$. Thus we get from Proposition 2.10 that there exists a semi-resolving set for points in $\Gamma_{n, q}$ of size

$$
\begin{aligned}
r_{\mathcal{P}}(n, q) & =2(q+1) \frac{q^{n}-q^{2}}{q^{2}-1}-2 q \frac{q^{n-2}-q^{2}}{q^{2}-1} \\
& =2 q^{n-1}+2 q^{n-2}+2\left(q^{n-4}+q^{n-6}+\cdots+q^{2}\right) .
\end{aligned}
$$


Now we turn to semi-resolving sets for lines. Let us start with a simple, but very useful observation.

Lemma 2.12. Let $\Sigma$ be a hyperplane in $\mathrm{PG}(n, q), \mathcal{S}$ be a semi-resolving set for points in $\Sigma$ and $\ell_{1}$ and $\ell_{2}$ be two distinct lines in $\mathrm{PG}(n, q)$. Suppose that none of the lines is contained in $\Sigma$ and the points $P_{1}=\Sigma \cap \ell_{1}$ and $P_{2}=\Sigma \cap \ell_{2}$ are distinct. Then the lines $\ell_{1}$ and $\ell_{2}$ are divided by $\mathcal{S}$ in $\Gamma_{n, q}$.

Proof. Since $\mathcal{S}$ is a semi-resolving set for points in $\Sigma$, there exists at least one element $s \in \mathcal{S}$ so that $d\left(P_{1}^{1}, s\right) \neq d\left(P_{2}^{1}, s\right)$. Hence

$$
d\left(\ell_{1}, s\right)=d\left(P_{1}^{1}, s\right)+1 \neq d\left(P_{2}^{1}, s\right)+1=d\left(\ell_{2}, s\right)
$$

the statement follows.

Theorem 2.13. For all $n>3$ and $q \geq 2 n-1$ there exists a semi-resolving set for lines in $\Gamma_{n, q}$ of size $r_{\mathcal{L}}(n, q)=2 n r_{\mathcal{P}}(n-1, q)$, where $r_{\mathcal{P}}$ follows (2.1) or (2.2) depending on the parity of $n-1$.

Proof. Let $\mathcal{H}=\left\{\Sigma_{1}, \Sigma_{2}, \ldots, \Sigma_{2 n}\right\}$ be a subset of $2 n$ hyperplanes of the $(q+1)$-element set formed by the dual hyperplanes of points on a normal rational curve. Then these hyperplanes are in general position, no $n+1$ of them have a point in common. Let $\mathcal{S}^{i}$ be a semi-resolving set for points in $\Sigma_{i}$. We claim that $\mathcal{S}=\bigcup_{i=1}^{2 n} \mathcal{S}^{i}$ is a semi-resolving set for lines in $\Gamma_{n, q}$.

Let $\ell_{1}$ and $\ell_{2}$ be two distinct lines in $\operatorname{PG}(n, q)$. We may assume that $\ell_{j}$ is contained in the intersection of $m_{j}$ elements of $\mathcal{H}$ for $j=1,2$, and $m_{1} \geq m_{2}$. The elements of $\mathcal{H}$ are in general position, so $n-1 \geq m_{j}$, hence $2 n-m_{1}-m_{2} \geq 2$. We may assume without loss of generality that $\ell_{j}$ intersects $\Sigma_{i}$ in a single point, denoted by $P_{j}^{i}$, for $i=$ $1,2, \ldots, 2 n-m_{1}-m_{2}$ and $j=1,2$. It could happen, that $P_{1}^{i_{1}}=P_{1}^{i_{2}}=\cdots=P_{1}^{i_{k}}$ for some indices, but $k \leq n-m_{2}$, otherwise the point would be a common point of at least $m_{1}+\left(n-m_{2}+1\right)>n$ elements of $\mathcal{H}$. So we may assume that $P_{1}^{1} \neq P_{1}^{2}$. As $\ell_{2}$ contains at most one point of $\ell_{1}$, we may also assume that $P_{1}^{1}$ is not on $\ell_{2}$. Then, by Lemma 2.12, $\ell_{1}$ and $\ell_{2}$ are divided by $\mathcal{S}^{1}$.

By Theorems 2.7 and 2.11, the size of $\mathcal{S}$ is at most $2 n r_{\mathcal{P}}(n-1, q)$ for $n>3$, thus the theorem is proved.

The union of a semi-resolving set for points and a semi-resolving set for lines is a resolving set. Thus Theorems 2.7, 2.11 and 2.13 give our first general upper bound.

Corollary 2.14. For all $n>3$ and $q \geq 2 n-1$ there exists a resolving set in $\Gamma_{n, q}$ of size

$$
r(n, q)=2 q^{n-1}+\left(4 n+1+(-1)^{n}\right) q^{n-2}+g_{n}(q),
$$

where $g_{n}$ is a polynomial of degree $n-3$ whose coefficients depend only on $n$.

In this bound the coefficient of the second highest degree term depends on the dimension. In the next part, by a more sophisticated construction, we prove an upper bound in which the coefficient of the second highest degree term is a constant. 
Proposition 2.15. Let $q=p^{h}$, p prime. Suppose that there exists a resolving set $\mathcal{S}_{3}$ in $\Gamma_{3, q}$ of size $2 q^{2}+a q+g_{3}(p)$, where $a \in \mathbb{R}, g_{3}$ is a polynomial of degree $s \leq h-1$, and $\mathcal{S}_{3}$ contains the $2 q^{2}+2$ elements of two disjoint line spreads. Then there exists a resolving set $\mathcal{S}_{4 m+3}$ in $\Gamma_{4 m+3, q}$ of size

$$
2 q^{n-1}+a q^{n-2}+g_{4 m+3}(p)
$$

where $g_{4 m+3}$ is a polynomial of degree at most $(n-3) h+s$.

Proof. As $(4 m+3)+1$ is divisible by $3+1$, there exists a 3 -spread in $\mathrm{PG}(4 m+3, q)$. This 3 -spread contains $t=\frac{q^{4(m+1)}-1}{q^{4}-1}$ elements, say $\Sigma_{3}^{1}, \Sigma_{3}^{2}, \ldots, \Sigma_{3}^{t}$, each of them is isomorphic to $\mathrm{PG}(3, q)$. By the assumption of the theorem, in each $\Sigma_{3}^{i}$ there exists a resolving set $\mathcal{S}_{3}^{i}$ of size $2 q^{2}+a q+g_{3}(p)$. We claim that

$$
\mathcal{S}=\bigcup_{i=1}^{t} \mathcal{S}_{3}^{i}
$$

is a resolving set in $\Gamma_{4 m+3, q}$. The elements of any pair of points and any point-line pair are obviously divided by $\mathcal{S}$. Let $\ell_{1}$ and $\ell_{2}$ be two lines. If at least one of them is contained in a $\Sigma_{3}^{i}$, then they are divided by $\mathcal{S}_{3}^{i}$. If none of them is contained in any $\Sigma_{3}^{i}$, then we may assume without loss of generality that $\ell_{1} \cap \Sigma_{3}^{1}$ is a point $P$ which is not on $\ell_{2}$. Let $s_{1}$ and $s_{2}$ be the two elements of the disjoint line spreads in $\mathcal{S}_{3}^{1}$ which are incident with $P$. Then $d\left(\ell_{1}, s_{1}\right)=d\left(\ell_{1}, s_{2}\right)=2$. As $\ell_{2}$ is not contained in $\mathcal{S}_{3}^{1}$, it cannot intersect both $s_{1}$ and $s_{2}$. Hence at least one of the distances $d\left(\ell_{2}, s_{1}\right)$ and $d\left(\ell_{2}, s_{2}\right)$ is 4 . Thus $\ell_{1}$ and $\ell_{2}$ are divided by $\mathcal{S}_{3}^{1} \subset \mathcal{S}$.

The size of $\mathcal{S}$ is

$$
\left(2 q^{2}+a q+g_{3}(p)\right) \frac{q^{n+1}-1}{q^{4}-1}=2 q^{n-1}+a q^{n-2}+g_{4 m+3}(p),
$$

where the degree of $g_{4 m+3}$ is $(n-3) h+\operatorname{deg} g_{4 m+3}=(n-3) h+s \leq(n-2) h-1$, so we are done.

Theorem 2.16. Let $q=p^{h}$, p prime. Suppose that there exists a resolving set in $\Gamma_{3, q}$ of size $2 q^{2}+a q+g_{3}^{3}(p)$ where $g_{3}^{3}$ is a polynomial of degree $s \leq h-1$. Then for $n \geq 3$ there exists a resolving set in $\Gamma_{n, q}$ of size

$$
r(n, q)=\left\{\begin{array}{lll}
2 q^{n-1}+(a+2) q^{n-2}+g_{n, 0}(p), & \text { if } n \equiv 0 & (\bmod 4), \\
2 q^{n-1}+(a+2) q^{n-2}+g_{n, 1}(p), & \text { if } n \equiv 1 & (\bmod 4), \\
2 q^{n-1}+(a+4) q^{n-2}+g_{n, 2}(p), & \text { if } n \equiv 2 & (\bmod 4), \\
2 q^{n-1}+a q^{n-2}+g_{n, 3}(p), & \text { if } n \equiv 3 & (\bmod 4),
\end{array}\right.
$$

where $g_{n, i}(i=0,1,2,3)$ is a polynomial of degree $(n-3) h+s$ whose coefficients depend only on $n$.

Proof. We prove it by induction on the dimension modulo 4 . For $n \equiv 3(\bmod 4)$ the statement follows from Proposition 2.15.

If $n \equiv 0(\bmod 4)$, then we apply Proposition 2.10 for $d=n-1$ with $k=r_{\mathcal{P}}(n-1, q)$ and $m=0$. Therefore by the induction hypothesis

$$
\begin{aligned}
r_{\mathcal{P}}(n, q) & \leq(q+1) r_{\mathcal{P}}(n-1, q)+\frac{q^{n-2}-1}{q-1} \\
& =2 q^{n-1}+(a+2) q^{n-2}+(q+1) g_{n-1,3}(p)+(a+1) q^{n-3}+q^{n-4}+\cdots+1
\end{aligned}
$$


Thus $g_{n, 0}(p)=(q+1) g_{n-1,3}(p)+(a+1) q^{n-3}+q^{n-4}+\cdots+1$, hence its degree is $(n-3) h+s \leq(n-2) h-1$.

If $n \equiv 1(\bmod 4)$, then $n-2 \equiv 3(\bmod 4)$, hence we can apply Proposition 2.10 for $d=n-1$ so that $\Sigma_{d-1}$ contains a resolving set constructed in Proposition 2.15. Then $k=2 q^{n-2}+(a+2) q^{n-3}+g_{n-1,0}(p)$ and $m=2 q^{n-3}+a q^{n-4}+g_{n-2,3}(p)$. Hence

$$
r_{\mathcal{P}}(n, q)=(q+1) k-q m+\frac{q^{n-2}-1}{q-1}=2 q^{n-1}+(a+2) q^{n-2}+g_{n, 1}(p),
$$

where

$$
g_{n, 1}(p)=(q+1) g_{n-1,0}(p)-q g_{n-2,3}(p)+3 q^{n-3}+q^{n-4}+\cdots+1,
$$

so its degree is $(n-3) h+s \leq(n-2) h-1$.

Finally, if $n \equiv 2(\bmod 4)$, then $n-3 \equiv 3(\bmod 4)$. Hence we cannot do better than apply Proposition 2.10 for $d=n-1$ so that $\Sigma_{d-1}$ contains entirely only elements of a $(d-2)$-dimensional resolving set constructed in Proposition 2.15. Now $k=2 q^{n-2}+(a+$ 2) $q^{n-3}+g_{n-1,1}(p)$ and $m=2 q^{n-4}+a q^{n-5}+g_{n-3,3}(p)$. This gives

$$
r_{\mathcal{P}}(n, q)=(q+1) k-q m+\frac{q^{n-2}-1}{q-1}=2 q^{n-1}+(a+4) q^{n-2}+g_{n, 2}(p),
$$

where

$$
g_{n, 2}(p)=(q+1) g_{n-1,1}(p)-q g_{n-3,3}(p)+(a+1) q^{n-3}+q^{n-4}+\cdots+1,
$$

thus its degree is $(n-3) h+s \leq(n-2) h-1$ again. The theorem is proved.

Let us remark that the polynomials $g_{n, i}$ can be determined exactly. We omit the long, but straightforward calculations, because their coefficients do not play any role in the rest of the paper.

In the next part of the section semi-resolving sets for lines in $\Gamma_{n, q}$ are investigated. In their constructions double blocking sets and their duals play an important role. For the relevant definitions and estimates on their sizes we refer to the paper of Ball and Blokhuis [3].

Theorem 2.17. For all $q>3$ there exists a semi-resolving set for lines in $\Gamma_{3, q}$ of size

$$
r_{\mathcal{L}}(3, q)=\min \left\{12 q-22,4 \tau_{2}(q)-10\right\},
$$

where $\tau_{2}(q)$ denotes the size of the smallest minimal double blocking set in $\mathrm{PG}(2, q)$.

Proof. First, we construct two sets of lines in $\operatorname{PG}(2, q)$ which are semi-resolving sets for points.

1. Let $E_{1}, E_{2}$, and $E_{3}$ be the vertices of a triangle, $\ell_{i}$ denote the line $E_{j} E_{k}$ and $\mathcal{P}_{i}$ be the pencil of lines with carrier $E_{i}$. Let

$$
\mathcal{S}=\mathcal{P}_{1} \cup \mathcal{P}_{2} \cup \mathcal{P}_{3} \backslash\left\{\ell_{1}, \ell_{2}, \ell_{3}, \ell\right\},
$$

where $\ell \in \mathcal{P}_{1}, \ell_{2} \neq \ell \neq \ell_{3}$. Then $\mathcal{S}$ is a semi-resolving set for points in $\Gamma_{2, q}$, because $U=\ell \cap \ell_{1}$ is a unique uncovered point, every point in the set $\ell_{1} \cup \ell_{2} \cup$ $\ell_{3} \backslash\left\{E_{1}, E_{2}, E_{3}, U\right\}$ is 1 -covered and all other points are at least 2-covered, hence resolved. The size of $\mathcal{S}$ is $3 q-4$. 
2. Let $\mathcal{D}$ be a dual double blocking set in $\operatorname{PG}(2, q)$. Then, by definition, each point is incident with at least two lines of $\mathcal{D}$. Thus if we delete an arbitrary line $\ell$ from $\mathcal{D}$, then the set of lines $\mathcal{D} \backslash\{\ell\}$ is still a semi-resolving set for points and, by the Principle of Duality, its size is at most $\tau_{2}(q)-1$.

Hence, for all $q>3$ there is a set of lines in $\operatorname{PG}(2, q)$ of size $\min \{3 q-4$, $\left.\tau_{2}(q)-1\right\}$ which is a semi-resolving set for points. Let $\mathcal{H}_{1}, \mathcal{H}_{2}, \mathcal{H}_{3}$, and $\mathcal{H}_{4}$ be the faces of a tetrahedron $\mathcal{K}$ in $\operatorname{PG}(3, q)$. Let $\mathcal{T}^{i}$ be a semi-resolving set for points in $\mathcal{H}_{i}$ which consists of lines only. We can choose $\mathcal{T}^{i}$ so that each edge of $\mathcal{K}$ belongs to both corresponding semi-resolving sets, because the full collineation group of $\operatorname{PG}(2, q)$ acts transitively on triangles. We claim that $\mathcal{S}=\cup_{i=1}^{4} \mathcal{T}^{i}$ is a semi-resolving set for lines in $\Gamma_{3, q}$.

The edges of $\mathcal{K}$ belong to $\mathcal{S}$, thus they are resolved by definition. Let $\ell_{1}$ and $\ell_{2}$ be lines such that none of them is an edge of $\mathcal{K}$. Then each of them is contained in at most one face of $\mathcal{K}$, so we may assume without loss of generality that $\ell_{1}$ intersects $\mathcal{H}_{i}$ in a single point, denoted by $P_{1}^{i}$, for $i=1,2,3$. We distinguish two main cases.

1. If $P_{1}^{1}=P_{1}^{2}=P_{1}^{3}$, then this point is a vertex $K$ of $\mathcal{K}$.

- If $\ell_{2}$ also contains $K$, then $\mathcal{H}_{4} \cap \ell_{1} \neq \mathcal{H}_{4} \cap \ell_{2}$, hence, by Lemma 2.12, the two lines are divided by $\mathcal{T}^{4}$.

- If $\ell_{2}$ does not contain $K$, then we may assume that $\mathcal{H}_{2} \cap \ell_{2}$ is a single point $P_{2}^{2}$. Since $P_{2}^{2} \neq K$, by Lemma 2.12, the two lines are divided by $\mathcal{T}^{2}$.

2. If none of $\ell_{1}$ and $\ell_{2}$ contains any vertex, then we may assume that $P_{1}^{1} \neq P_{1}^{2}$.

- If $\ell_{2}$ is not contained in neither $\mathcal{H}_{1}$ nor $\mathcal{H}_{2}$, then it intersects $\mathcal{H}_{i}$ in a single point, denoted by $P_{2}^{i}$, for $i=1,2$. Since $\ell_{1} \cap \ell_{2}$ contains at most one point, we may assume that $P_{1}^{1} \neq P_{2}^{1}$. Then, by Lemma 2.12, the two lines are divided by $\mathcal{T}^{1}$.

- Finally, if $\ell_{2}$ is contained in one of $\mathcal{H}_{1}$ and $\mathcal{H}_{2}$, then we may assume that $\ell_{2} \subset \mathcal{H}_{1}$ and $\ell_{2} \cap \mathcal{H}_{2}$ in a single point $P_{2}^{2}$. Then $P_{2}^{2}$ is in $\mathcal{H}_{1}$, so $P_{2}^{2} \neq P_{1}^{2}$, because otherwise $\ell_{1} \subset \mathcal{H}_{1}$. Hence, by Lemma 2.12, the two lines are divided by $\mathcal{T}^{2}$.

Since $\mathcal{S}$ has the required size, we are done.

Remark 2.18. Let us remark that if the double blocking set $\mathcal{D}$ in the proof of Theorem 2.17 is the disjoint union of two dual blocking sets, then not only one, but two lines can be deleted without violating the semi-resolving set property. We will consider this case in Section 3, Theorem 3.1.

Unfortunately, the exact value of $\tau_{2}(q)$ is not known in general. It is known that $\tau_{2}(q)=$ $2 q+2 \sqrt{q}+2$ for $q$ is a square and $q>16$ [3, Theorem 3.1], and for some small values of $q$. In the latter case for the known values $\tau_{2}(q)>3 q-3$ always holds. Combining the semi-resolving set for points constructed in Corollary 2.7 and the semi-resolving set for lines of Theorem 2.17, we get the following upper bound on $\mu\left(\Gamma_{3, q}\right)$.

Theorem 2.19. The metric dimension of $\Gamma_{3, q}$ satisfies the inequality

$$
\mu\left(\Gamma_{3, q}\right) \leq 2 q^{2}+12 q-24
$$

for all $q>3$. 
Proof. For $q>3$ let $\mathcal{S}_{\mathcal{L}}$ be a semi-resolving set for lines of size $12 q-22$ constructed in Theorem 2.17. Let $\mathcal{L}_{1}$ be a regular line spread of $\operatorname{PG}(3, q)$ which contains two skew (non-intersecting) elements of $\mathcal{S}_{\mathcal{L}}$. Such spread exists, because the collineation group of $\operatorname{PG}(3, q)$ acts transitively on the pairs of skew lines. Create a semi-resolving set for points $\mathcal{S}_{\mathcal{P}}$ which contains $\mathcal{L}_{1}$ as we did it in Corollary 2.7. Then $\mathcal{S}=\mathcal{S}_{\mathcal{L}} \cup \mathcal{S}_{\mathcal{P}}$ is a resolving set in $\Gamma_{3, q}$ and its size is $2 q^{2}+12 q-24$. This proves the inequality.

By combining Theorem 2.16, with $s=0$, and Theorem 2.19, we get the following bounds.

Corollary 2.20. Let $n \geq 3$ and $q>3$. Then the metric dimension of $\Gamma_{n, q}$ satisfies the inequality

$$
\mu\left(\Gamma_{n, q}\right) \leq \begin{cases}2 q^{n-1}+14 q^{n-2}+h_{n, 2}(q), & \text { if } n \equiv 0 \text { or } n \equiv 1 \quad(\bmod 4) \\ 2 q^{n-1}+16 q^{n-2}+h_{n, 3}(q), & \text { if } n \equiv 2 \quad(\bmod 4) \\ 2 q^{n-1}+12 q^{n-2}+h_{n, 1}(q), & \text { if } n \equiv 3 \quad(\bmod 4)\end{cases}
$$

where $h_{n, i}(i=1,2,3)$ is a polynomial of degree at most $n-3$ whose coefficients depend only on $n$.

The metric dimension of $\Gamma_{2, q}$ for $q \geq 23$ was determined by Héger and Takáts [12]. For higher dimensions we do not know the exact value, but Theorems 2.1, 2.19, and Corollary 2.20 imply the following result.

Corollary 2.21. For all $n>2$ and $q>3$

$$
\left|\mu\left(\Gamma_{n, q}\right)-2 q^{n-1}\right|=O\left(q^{n-2}\right) .
$$

This means that $\mu\left(\Gamma_{n, q}\right)$ is asymptotically $2 q^{n-1}$. The number of vertices in $\Gamma_{n, q}$ is $v=\frac{q^{n+1}-1}{q-1}+\frac{\left(q^{n+1}-1\right)\left(q^{n}-1\right)}{(q+1)(q-1)^{2}}$, so its metric dimension is roughly $2 \sqrt{v}$. The automorphism group of $\operatorname{PG}(n, q)$ is $\operatorname{P\Gamma L}(n+1, q)$ and it is well-known that its base size is $n+1$ if $q$ is a prime, and it is $n+2$ if $q=p^{h}$ with $h>1$. Hence the dimension jump of $\Gamma_{n, q}$ is roughly $2 \sqrt{v}$.

\section{Bounds for $q=p^{h}, h \geq 2$}

In this section we consider the case $q=p^{h}, h>1$. In the case $h$ even, we will present a better bound on the size of a semi-resolving set for points in $\Gamma_{3, q}$ using small dual double blocking sets in $\operatorname{PG}(2, q)$. When $h>2$, then we will show that a particular type of spread of lines in $\mathrm{PG}(3, q)$ can be used to resolve the lines. In fact, for a regular spread, there exist many pairs of lines of the spaces intersecting the same set of elements of the spread. We now investigate a different type of spread, called aregular, and we determine all the lines of the space intersecting the same set of elements of the spread; see Theorem 3.6. The main goal is to construct a set of lines of $\operatorname{PG}(3, q)$ which resolves all the lines of the spaces; see Theorem 3.7.

Theorem 3.1. If $q$ is a square, then the metric dimension of $\Gamma_{3, q}$ satisfies the inequality

$$
\mu\left(\Gamma_{3, q}\right) \leq 2 q^{2}+8 q+8 \sqrt{q}-8
$$


Proof. The union of the sets of lines of two disjoint Baer subplanes is a dual double blocking set in $\operatorname{PG}(2, q)$ and its size is $2 q+2 \sqrt{q}+2$. This set is the disjoint union of two dual blocking sets. Hence, by a result of Héger and Takáts [12, Proposition 22], we can delete two of its lines so that the remaining set is still a semi-resolving set for points in $\operatorname{PG}(2, q)$; see also Remark 2.18.

Thus we can construct a semi-resolving set for lines $\mathcal{S}_{\mathcal{L}}$ of size $8 q+8 \sqrt{q}-6$ by the method applied in the proof of Theorem 2.17. Finally, we can extend it to a resolving set of size $2 q^{2}+8 q+8 \sqrt{q}-8$ in the same way as we did in the proof of Theorem 2.19. bounds.

By combining Theorem 2.16, with $s=0$, and Theorem 2.19, we get the following

Corollary 3.2. If $q$ is a square and $n \geq 3$, then the metric dimension of $\Gamma_{n, q}$ satisfies the inequality

$$
\mu\left(\Gamma_{n, q}\right) \leq \begin{cases}2 q^{n-1}+10 q^{n-2}+h_{n, 2}(q), & \text { if } n \equiv 0 \text { or } 1 \quad(\bmod 4), \\ 2 q^{n-1}+12 q^{n-2}+h_{n, 3}(q), & \text { if } n \equiv 2 \quad(\bmod 4), \\ 2 q^{n-1}+8 q^{n-2}+h_{n, 1}(q), & \text { if } n \equiv 3 \quad(\bmod 4)\end{cases}
$$

where $h_{n, i}(i=1,2,3)$ is a polynomial of degree at most $n-3$ whose coefficients depend only on $n$.

Theorem 3.3 ([13, Theorem 17.3.3]). Let $q=p^{h}, h>1$, and choose $b, c \in \mathbb{F}_{q}^{*}$ such that the polynomial $t^{p+1}-t b+c$ has no roots in $\mathbb{F}_{q}$. Let

$$
\mathcal{A}_{b, c}=\left\{t_{\alpha, \beta}: \alpha, \beta \in \mathbb{F}_{q}\right\} \cup\{Z=T=0\},
$$

where $t_{\alpha, \beta}$ is the line through the points $(\alpha: \beta: 1: 0)$ and $\left(c \beta^{p}: \alpha^{p}+b \beta^{p}: 0: 1\right)$. Then $\mathcal{A}_{b, c}$ is a spread, called the aregular spread.

In what follows, we will associate to each line $r$ of the space an algebraic curve $\mathcal{C}_{r}: F_{r}(X, Y, T)=0$ such that $t_{\alpha, \beta}$ intersects $r$ if and only if $F_{r}(\alpha, \beta, 1)=0$. We distinguish four types of lines.

1. Lines $r_{x, y, \ell, m}$ through the points $(x: y: 0: 1)$ and $(\ell: m: 1: 0)$. Note that if $x=c m^{p}$ and $y=\ell^{p}+b m^{p}$ then $r_{x, y, \ell, m}$ coincides with $t_{\ell, m} \in \mathcal{A}_{b, c}$. From now on we consider $(x, y) \neq\left(\mathrm{cm}^{p}, \ell^{p}+b m^{p}\right)$. A line $t_{\alpha, \beta}$ intersects $r=r_{x, y, \ell, m}$ if and only if for some $\lambda \in \mathbb{F}_{q}$ the points

$$
(x+\lambda \ell: y+\lambda m: \lambda: 1), \quad(\alpha: \beta: 1: 0), \quad\left(c \beta^{p}: \alpha^{p}+b \beta^{p}: 0: 1\right)
$$

are collinear, that is

$$
x+\lambda \ell-c \beta^{p}=\lambda \alpha, \quad y+\lambda m-\left(\alpha^{p}+b \beta^{p}\right)=\lambda \beta .
$$

This implies

$$
\lambda(\ell-\alpha)=c \beta^{p}-x, \quad \lambda(m-\beta)=\alpha^{p}+b \beta^{p}-y,
$$

and therefore

$$
\left(c \beta^{p}-x\right)(m-\beta)=\left(\alpha^{p}+b \beta^{p}-y\right)(\ell-\alpha) .
$$

In this case, $F_{r}(X, Y, T)=\left(c Y^{p}-x T^{p}\right)(m T-Y)-\left(X^{p}+b Y^{p}-y T^{p}\right)(\ell T-X)$. 
2. Lines $s=s_{x, y, z, \ell}$ through the points $(x: y: z: 1)$ and $(\ell: 1: 0: 0)$. A line $t_{\alpha, \beta}$ intersects $s_{x, y, z, \ell}$ if and only if for some $\lambda \in \mathbb{F}_{q}$ the points

$$
(x+\lambda \ell: y+\lambda: z: 1), \quad(\alpha: \beta: 1: 0), \quad\left(c \beta^{p}: \alpha^{p}+b \beta^{p}: 0: 1\right)
$$

are collinear, that is

$$
x+\lambda \ell-c \beta^{p}=z \alpha, \quad y+\lambda-\left(\alpha^{p}+b \beta^{p}\right)=z \beta .
$$

This implies

$$
\lambda \ell=-x+c \beta^{p}+z \alpha, \quad \lambda=-y+z \beta+\alpha^{p}+b \beta^{p}
$$

and therefore

$$
-x+c \beta^{p}+z \alpha=\ell\left(-y+z \beta+\alpha^{p}+b \beta^{p}\right) .
$$

So, $F_{s}(X, Y, T)=-\ell X^{p}+(c-\ell b) Y^{p}+z X T^{p-1}-\ell z Y T^{p-1}+(\ell y-x) T^{p}$.

3. Lines $u=u_{x, y, z}$ through the points $(x: y: z: 1)$ and $(1: 0: 0: 0)$. In this case, $F_{u}(X, Y, T)=X^{p}+b Y^{p}+z Y T^{p-1}-y T^{p}$.

4. Lines $v=v_{x, y, z}$ contained in the planes $T=0$ and $x X+y Y+z Z=0$. Then, $F_{v}(X, Y, T)=x X+y Y+z T$.

Such a curve is absolutely irreducible if $z \neq 0$, otherwise it collapses into a single line.

Proposition 3.4. Consider the curves $\mathcal{C}_{r}, \mathcal{C}_{s}, \mathcal{C}_{u}$, and $\mathcal{C}_{v}$. Then

1. $\mathcal{C}_{r}$ is absolutely irreducible;

2. $\mathcal{C}_{s}$ is either absolutely irreducible or a line repeated $p$ times;

3. $\mathcal{C}_{u}$ is either absolutely irreducible or a line repeated $p$ times.

\section{Proof.}

1. Now we prove that $\mathcal{C}_{r}$ is absolutely irreducible. Let $\varphi(X, Y, T)=\left(X+x_{0} T, Y+\right.$ $\left.y_{0} T, T\right)$ with $x_{0}^{p}=y-b x / c, y_{0}^{p}=x / c$. Then

$$
\begin{aligned}
F_{r}(\varphi(X, Y, T)) \\
=\left(c\left(Y+y_{0} T\right)^{p}-x T^{p}\right)\left(m T-Y-y_{0} T\right) \\
\quad-\left(\left(X+x_{0} T\right)^{p}+b\left(Y+y_{0} T\right)^{p}-y T^{p}\right)\left(\ell T-X-x_{0} T\right) \\
=\left(c Y^{p}+c y_{0}^{p} T^{p}-x T^{p}\right)\left(m T-Y-y_{0} T\right) \\
\quad-\left(X^{p}+x_{0}^{p} T^{p}+b Y^{p}+y_{0}^{p} T^{p}-y T^{p}\right)\left(\ell T-X-x_{0} T\right) \\
=\left(c Y^{p}+x T^{p}-x T^{p}\right)\left(m T-Y-y_{0} T\right) \\
\quad-\left(X^{p}+y T^{p}-b x / c T^{p}+b Y^{p}+b x / c T^{p}-y T^{p}\right)\left(\ell T-X-x_{0} T\right) \\
=c Y^{p}\left(m T-Y-y_{0} T\right)-\left(X^{p}+b Y^{p}\right)\left(\ell T-X-x_{0} T\right) \\
=G_{r}(X, Y, T) .
\end{aligned}
$$

Finally

$$
G_{r}(X, 1, Y)=c\left(m Y-1-y_{0} Y\right)-\left(X^{p}+b\right)\left(\ell Y-X-x_{0} Y\right),
$$


that is the curve $\mathcal{C}_{r}$ is $\mathbb{F}_{q}$-isomorphic to

$$
\mathcal{C}^{\prime}: Y=\frac{c-X\left(X^{p}+b\right)}{c m-c y_{0}+\left(x_{0}-\ell\right)\left(X^{p}+b\right)},
$$

which is an irreducible rational curve with $q+1 \mathbb{F}_{q}$-rational points (note that $(x, y) \neq$ $\left(c m^{p}, \ell^{p}+b m^{p}\right)$ yields $m \neq y_{0}$ or $\left.\ell \neq x_{0}\right)$. This means that the curve $\mathcal{C}_{r}$ is absolutely irreducible.

2. First, note that the homogeneous term $-\ell X^{p}+(c-\ell b) Y^{p}$ cannot vanish otherwise $c=0$, a contradiction.

- If $(\ell, z)=(0,0), \mathcal{C}_{s}$ is a line of type $b_{0} Y+c_{0} T=0$ repeated $p$ times.

- If $\ell=0$ and $z \neq 0$, then $F_{s}(X, Y, T)$ reads $c Y^{p}+z X T^{p-1}-x T^{p}$ and $\mathcal{C}_{s}$ is absolutely irreducible.

- If $\ell \neq 0$ and $z=0$ then $\mathcal{C}_{s}$ is a (repeated) line $a_{0} X+b_{0} Y+c_{0} T=0$, where $a_{0}^{p}=-\ell, b_{0}^{p}=(c-\ell b), c_{0}^{p}=(\ell y-x)$.

- If $\ell \neq 0$ and $z \neq 0$ then consider $\varphi(X, Y, T)=(X+\sqrt[p]{(c-\ell b) / \ell} Y, Y, T)$ and so

$$
\begin{aligned}
& G_{s}(X, Y, T)=F_{s}(\varphi(X, Y, T))= \\
& \quad-\ell X^{p}+z X T^{p-1}+z(\sqrt[p]{(c-\ell b) / \ell}-\ell) Y T^{p-1}+(\ell y-x) T^{p} .
\end{aligned}
$$

By our assumption of $b, c$, there is no $\ell \in \mathbb{F}_{q}$ such that $\sqrt[p]{(c-\ell b) / \ell}-\ell=0$. The curve $G_{s}(X, Y, T)=0$ is rational and irreducible and it is $\mathbb{F}_{q}$-isomorphic to $\mathcal{C}_{s}$.

3. Clear.

Proposition 3.5. Let $q=p^{h}, h>2$. Two lines of the same type $(r, s, u, v)$ do not intersect the same set of lines of the aregular spread $\mathcal{A}_{b, c}$.

Proof. The assumption $h>2$ implies $q+1>(p+1)^{2}$. The curves $\mathcal{C}_{r}, \mathcal{C}_{s}, \mathcal{C}_{u}, \mathcal{C}_{v}$ have degree at most $p$ and they have $q+1 \mathbb{F}_{q}$-rational points (corresponding to the lines of the spread intersecting them). By Proposition 3.4, such curves are either absolutely irreducible or they consist of a repeated line. Thus, if two curves attached to the lines $w_{1}$ and $w_{2}$ of the same type share $q+1 \mathbb{F}_{q}$-rational points, the corresponding polynomials must be proportional. By direct computations, this yields $w_{1}=w_{2}$.

Theorem 3.6. Let $q=p^{h}, h>2$. If two lines in $\mathrm{PG}(3, q)$ intersect the same set of lines of the spread $\mathcal{A}_{b, c}$ then one of them lies on the plane $Z=0$ and the other on the plane $T=0$.

Proof. The reduced (absolutely irreducible) curves associated with the different types of lines $(r, s, u, v)$ have degree $p+1$, degree $p$ or 1 , degree $p$ or 1 , and degree 1 , respectively. They can share $q+1 \mathbb{F}_{q}$-rational points only in the following cases:

- both $\mathcal{C}_{s}$ and $\mathcal{C}_{u}$ have degree $p$;

- both $\mathcal{C}_{s}$ and $\mathcal{C}_{u}$ have degree 1 ;

- both $\mathcal{C}_{s}$ and $\mathcal{C}_{v}$ have degree 1 ; 
- both $\mathcal{C}_{u}$ and $\mathcal{C}_{v}$ have degree 1 .

The first case is not possible. The second case would imply $c=0$, a contradiction. Recall that the lines $v=v_{x, y, z}$ are contained in the plane $T=0$ of $\operatorname{PG}(3, q)$. The claim follows observing that if $\mathcal{C}_{s}$ or $\mathcal{C}_{u}$ have degree 1 , then $s=s_{x, y, 0, \ell}$ or $u=u_{x, y, 0}$. So, both $s$ and $u$ are contained in the plane $Z=0$.

Theorem 3.7. Let $q=p^{h}, h>2$. Then there exists a set of $q^{2}+3$ lines resolving all the lines of $\mathrm{PG}(3, q)$.

Proof. Consider the aregular spread $\mathcal{A}_{b, c}$ with $b, c \in \mathbb{F}_{q}^{*}$ and such that the polynomial $t^{p+1}-t b+c$ has no roots in $\mathbb{F}_{q}$. We already know by Theorem 3.6 that lines of $\operatorname{PG}(3, q)$ intersecting the same set of elements of $\mathcal{A}_{b, c}$ are contained in the planes $Z=0$ or $T=0$. Note that two lines in a fixed plane cannot intersect the same elements of $\mathcal{A}_{b, c}$. Consider two distinct extra lines $w_{1}$ and $w_{2}$ contained in $Z=0$ intersecting the line $Z=T=0$ at two distinct points. It is readily seen that $\mathcal{A}_{b, c} \cup\left\{w_{1}, w_{2}\right\}$ resolves all the lines of $\operatorname{PG}(3, q)$.

Corollary 3.8. If $q=p^{h}, h>2$, then there exists a resolving set in $\Gamma_{3, q}$ of size $2 q^{2}+2$.

Proof. Consider the set of $q^{2}+3$ lines from Theorem 3.7 and use the argument of Theorem 2.9. One of the two extra lines could be an element of the other spread. Finally, delete one line from the modified regular spread.

Finally, the following bounds are obtained combining again Theorem 2.16, with $s=0$, and Theorem 2.19.

Corollary 3.9. If $q=p^{h}, h>2$, then the metric dimension of $\Gamma_{n, q}$ satisfies the inequality

$$
\mu\left(\Gamma_{n, q}\right) \leq \begin{cases}2 q^{n-1}+2 q^{n-2}+h_{n, 2}(q), & \text { if } n \equiv 0 \text { or } 1 \quad(\bmod 4), \\ 2 q^{n-1}+4 q^{n-2}+h_{n, 3}(q), & \text { if } n \equiv 2 \quad(\bmod 4), \\ 2 q^{n-1}+h_{n, 1}(q), & \text { if } n \equiv 3 \quad(\bmod 4),\end{cases}
$$

where $h_{n, i}(i=1,2,3)$ is a polynomial of degree at most $n-3$ whose coefficients depend only on $n$.

\section{Resolving sets for small $q$}

We performed a computer search to obtain sets of lines that are semi-resolving sets for lines in $\mathrm{PG}(3, q)$ for small $q$. We used MAGMA, a computer algebra system for symbolic computation developed at the University of Sydney; see [6]. We started classifying all set of lines of a certain size $k$. Then we extended the non-equivalent sets of size $k$ using a backtracking algorithm.

In $\mathrm{PG}(3,2)$ there are 35 lines, so a semi-resolving set for lines must contain at least six elements. We found that there are 165 non-equivalent sets of lines of size six. Forty-eight of them are semi-resolving sets for lines in $\mathrm{PG}(3,2)$. An example is the following set of six lines:

$$
\begin{aligned}
& \{\langle(1: 0: 0: 0),(0: 1: 0: 0)\rangle,\langle(0: 1: 0: 0),(0: 0: 0: 1)\rangle, \\
& \quad\langle(0: 0: 1: 0),(0: 0: 0: 1)\rangle,\langle(0: 0: 0: 1),(1: 1: 0: 0)\rangle, \\
& \langle(0: 1: 0: 0),(1: 1: 1: 1)\rangle,\langle(1: 1: 0: 0),(0: 0: 1: 1)\rangle\} .
\end{aligned}
$$


In $\mathrm{PG}(3,3)$ there are 130 lines, so a semi-resolving set for lines must contain at least eight elements. We found that there are 10681 non-equivalent sets of lines of size seven. An exhaustive search by backtracking has proved that no set of lines of size eight or nine is a semi-resolving set for lines in $\mathrm{PG}(3,3)$. There exist semi-resolving sets for lines of size ten. An example is the following set of ten lines:

$$
\begin{aligned}
& \{\langle(1: 0: 0: 0),(0: 1: 0: 0)\rangle,\langle(1: 0: 0: 0),(0: 0: 0: 1)\rangle, \\
& \langle(0: 1: 0: 0),(0: 0: 1: 0)\rangle,\langle(0: 1: 0: 0),(1: 0: 0: 1)\rangle, \\
& \quad\langle(1: 0: 1: 2),(0: 1: 1: 0)\rangle,\langle(1: 0: 0: 2),(0: 0: 1: 1)\rangle, \\
& \langle(1: 0: 0: 0),(0: 1: 1: 2)\rangle,\langle(1: 0: 0: 0),(0: 1: 1: 0)\rangle, \\
& \quad\langle(0: 1: 1: 1),(1: 2: 0: 0)\rangle,\langle(1: 1: 1: 0),(0: 1: 2: 0)\rangle\} .
\end{aligned}
$$

\section{ORCID iDs}

Daniele Bartoli (iD https://orcid.org/0000-0002-5767-1679

György Kiss (D) https://orcid.org/0000-0003-3312-9575

Stefano Marcugini (iD https://orcid.org/0000-0002-7961-0260

Fernanda Pambianco (D) https://orcid.org/0000-0001-5476-5365

\section{References}

[1] J. André, Über nicht-Desarguessche Ebenen mit transitiver Translationsgruppe, Math. Z. 60 (1954), 156-186, doi:10.1007/bf01187370.

[2] R. F. Bailey and P. J. Cameron, Base size, metric dimension and other invariants of groups and graphs, Bull. Lond. Math. Soc. 43 (2011), 209-242, doi:10.1112/blms/bdq096.

[3] S. Ball and A. Blokhuis, On the size of a double blocking set in $\mathrm{PG}(2, q)$, Finite Fields Appl. 2 (1996), 125-137, doi:10.1006/ffta.1996.9999.

[4] D. Bartoli, T. Héger, Gy. Kiss and M. Takáts, On the metric dimension of affine planes, biaffine planes and generalized quadrangles, Australas. J. Combin. 72 (2018), 226-248, https: // ajc.maths.uq.edu.au/pdf/72/ajc_v72_p226.pdf.

[5] D. Bartoli, Gy. Kiss, S. Marcugini and F. Pambianco, Resolving sets for higher dimensional projective spaces, Finite Fields Appl. 67 (2020), 101723 (14 pages), doi:10.1016/j.ffa.2020. 101723.

[6] W. Bosma, J. Cannon and C. Playoust, The Magma algebra system I: The user language, J. Symbolic Comput. 24 (1997), 235-265, doi:10.1006/jsco.1996.0125.

[7] R. H. Bruck and R. C. Bose, The construction of translation planes from projective spaces, $J$. Algebra 1 (1964), 85-102, doi:10.1016/0021-8693(64)90010-9.

[8] T. Etzion, Partial $k$-parallelisms in finite projective spaces, J. Combin. Des. 23 (2015), 101-114, doi:10.1002/jcd.21392.

[9] S. L. Fancsali and P. Sziklai, Lines in higgledy-piggledy arrangement, Electron. J. Combin. 21 (2014), \#P2.56 (15 pages), doi:10.37236/4149.

[10] T. Héger, B. Patkós and M. Takáts, Search problems in vector spaces, Des. Codes Cryptogr. 76 (2015), 207-216, doi:10.1007/s10623-014-9941-9.

[11] T. Héger, P. Szilárd and M. Takáts, The metric dimension of the incidence graphs of projective planes of small order, Australas. J. Combin. 78 (2020), 352-375, https: / / a jc . maths. $\mathrm{uq} \cdot \mathrm{edu} \cdot \mathrm{au} / \mathrm{pdf} / 78 / \mathrm{ajc}$ _v78_p352.pdf. 
[12] T. Héger and M. Takáts, Resolving sets and semi-resolving sets in finite projective planes, Electron. J. Combin. 19 (2012), \#P30 (21 pages), doi:10.37236/2582.

[13] J. W. P. Hirschfeld, Finite Projective Spaces of Three Dimensions, Clarendon Press, Oxford, 1985.

[14] J. W. P. Hirschfeld, Projective Geometries over Finite Fields, Clarendon Press, Oxford, 2nd edition, 1998.

[15] J. W. P. Hirschfeld, G. Korchmáros and F. Torres, Algebraic Curves over a Finite Field, Princeton Series in Applied Mathematics, Princeton University Press, Princeton, 2008.

[16] Gy. Kiss and T. Szőnyi, Finite Geometries, CRC Press, Boca Raton, Florida, 2019, doi:10. 1201/9781315120072.

[17] B. Segre, Teoria di Galois, fibrazioni proiettive e geometrie non desarguesiane, Ann. Mat. Pura Appl. 64 (1964), 1-76, doi:10.1007/bf02410047.

[18] C. C. Sims, Determining the conjugacy classes of a permutation group, in: G. Birkhoff and M. Hall, Jr. (eds.), Computers in Algebra and Number Theory, American Mathematical Society, Providence, Rhode Island, volume IV of SIAM-AMS Proceedings, 1971 pp. 191-195, proceedings of a Symposium in Applied Mathematics of the American Mathematical Society and the Society for Industrial and Applied Mathematics, held in New York City, March 25 26, 1970. 